\title{
Dynamic Analysis of Helical Planetary Gear Sets under Combined Force and Moment Loading
}

\author{
Yanfang Liu, Junbin Lai, Peng Dong, and Xiangyang Xu \\ Beijing Key Laboratory for High Efficient Transmission and System Control of New Energy Resource Vehicle, \\ School of Transportation Science and Engineering, Beihang University, Beijing 100191, China
}

Correspondence should be addressed to Peng Dong; dongpengbeihang@163.com

Received 1 August 2017; Accepted 5 November 2017; Published 22 November 2017

Academic Editor: Tai Thai

Copyright (C) 2017 Yanfang Liu et al. This is an open access article distributed under the Creative Commons Attribution License, which permits unrestricted use, distribution, and reproduction in any medium, provided the original work is properly cited.

The dynamic behavior of a single-stage planetary gear set with helical gears of multishaft automotive automatic transmissions has been studied, in which one component of the planetary gear set is imposed by additional external vertical and axial loading from countershaft gear pair in addition to the moment. Under these combined loading conditions, the contributions of the deflections of the ring gear and the carrier cannot be neglected. A three-dimensional nonlinear time-variant dynamic model considering not only the transverse, torsional, axial, and rotational motions of the gears but also the elasticity of the mounted shafts has been developed by combining the lumped parameter method with finite element method. The natural modes and the forced vibration responses due to static transmission errors have been obtained. The proposed dynamic model is employed to describe the effects of the combined external loading condition and positioning on the dynamic behavior of a four-planet system.

\section{Introduction}

Planetary gear trains (PGTs) are the core component of the powertrain transmission systems used in many aerospace, vessel, automotive, construction machinery, and wind-power applications. Under dynamic conditions, the forces acting on gears are amplified, which would lead to larger dynamic loads and stresses and potentially reduce the fatigue life of gears. Furthermore, high-frequency alternating forces due to the dynamic behavior are transmitted to the radiating surfaces as excitation for gear whine. Therefore, dynamic analysis is essential in describing the vibration and noise and even durability of any geared power transmission systems. A dynamic model for predicting vibration amplitudes and dynamic forces is necessary to evaluate the noise and durability of a given gear system, which is helpful to identify potential problems and find capable solutions.

Lumped parameter method is widely used to develop the dynamic model of PGTs, in which the PGT is simplified as a topology structure by assuming each component as rigid and the connection through kinematical joints, springs, and dampers. Various models have been proposed by many researchers over the past decades. With respect to the number of freedoms, there are pure torsional vibration models [1], two-dimensional models [2], and three-dimensional models [3]. In terms of considering the stiffness changes and backlash or not, there are linear or nonlinear and time-invariant or time-variant dynamic models [1,4-6]. Those models can analyze the vibration characteristics, such as natural modes and forced vibration responses. Another unique behavior, namely, modulation sidebands for PGTs, is studied by a few researchers. Inalpolat and Kahraman [4] predicted modulation sidebands of a simple-stage planetary gear train by introducing the influence of manufacturing errors on the gear transmission error excitations into a nonlinear timevariant dynamic model. The inflexibility assumption of the gear system in the lumped parameter method deviates from the real elastic mechanical structure. Kubur et al. [7] proposed a dynamic model combining a three-dimensional discrete dynamic model of counter-shaft helical gear pairs with a finite element model of shaft structures. Abousleiman et al. [8] proposed a hybrid model for spur and helical gear planetary drives in which the contributions of the deflections of the ring gear and the carrier are introduced via substructures derived from 3D finite element methods while the sun gear is assumed to be rigid. 


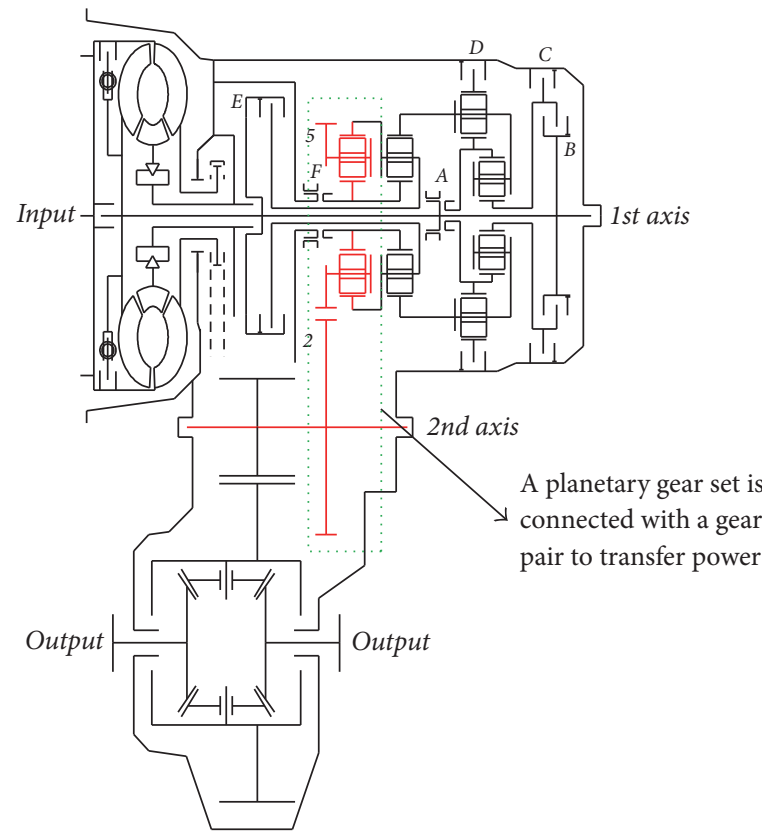

(a) ZF 9-speed AT [9]

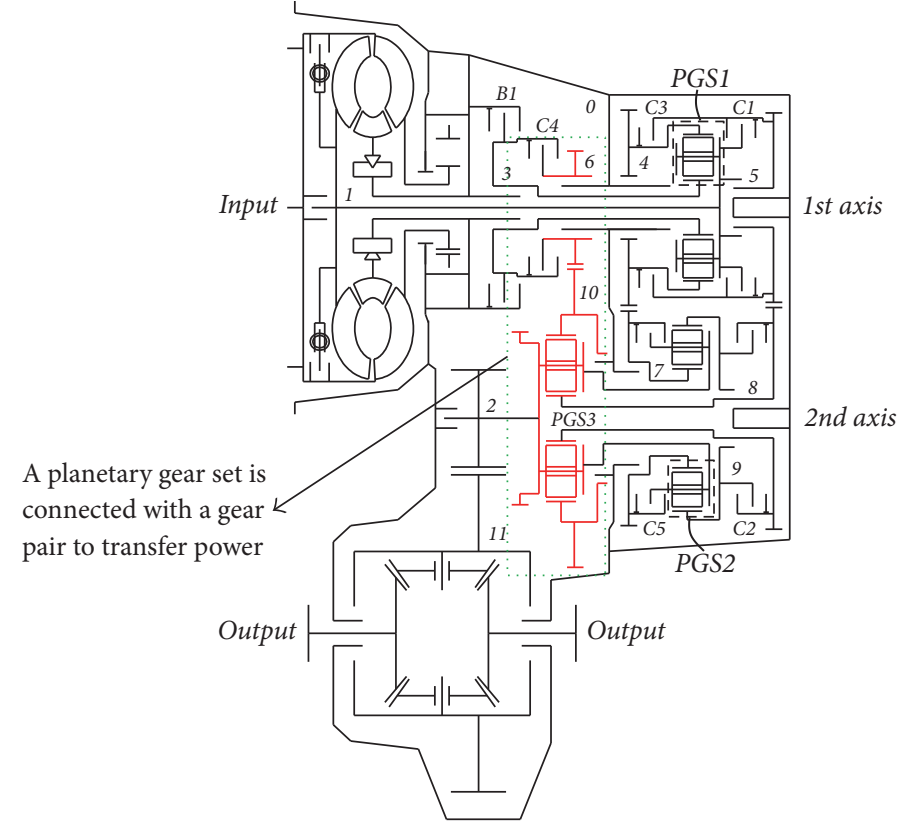

(b) ShengRui 8-speed AT [10]

FIGURE 1: Schemes of two FWD AT examples with parallel-axis layouts.

Furthermore, there are many new developed multispeed automatic transmission layouts in which planetary gear sets and gear pairs are assembled in parallel axes in recent ten years. Figure 1 shows two examples of this kind of structural schemes. This kind of structural layout is well suitable for front-wheel drive (FWD) vehicles. Only gear pairs are mounted on the counter-shaft as shown in Figure 1(a) while both planetary gear sets and gear pairs are mounted on the countershaft as presented in Figure 1(b). One gear of a gear pair is usually fixedly connected with one component of a planetary gear set. For example, the driving gear of the gear pair 5-2 in Figure 1(a) is fixedly connected with the carrier of the planetary gear set on the far left through shaft, and the driven gear of the gear pair 6-10 in Figure 1(b) is fixedly connected with the ring gear of the planetary gear set on the left side of the counter-shaft; the details are shown in the dotted box in Figure 1. When the power is transmitted through gear pairs, due to the gear tooth meshing forces, additional vertical forces and even axial forces are generated and transmitted to the supporting shafts of the gears. When one gear is connected with one component of a planetary gear set, the vertical and/or axial forces will be shared by the planetary gear set too, whose force environment is different from the traditional concentric automatic transmission based on multiple planetary gear sets. As for this kind of structure, the shafts of planetary gear sets imposed by vertical and/or axial forces in addition to torque would have much more deflection and thus influence the dynamic behavior of the gear system. So it is necessary to investigate the dynamic characteristics of this of kind gear system.

Without loss of generality, a single-stage PGT imposed by combined external vertical forces and/or axial forces in addition to the moment is introduced in the following. This study aims at developing a dynamic model of this kind of PGTs considering the flexibility of all components. As the focus is not only on the gears but also on other components, the model must have the capability of including the most relevant design parameters of gears, shafts, and bearings to quantify the direct influence of each of these parameters on the overall dynamic behavior. Based on the model, the dynamic behavior of PGTs under additional external vertical force and/or axial forces will be predicted and analyzed. Limited parameter studies will be performed to describe the effect of some of the key system parameters on the dynamic behavior. The intent of this model is to help designers obtain the most favorable configuration for the most desirable dynamic behavior at any stage of the development of the gear system.

\section{Dynamic Model}

2.1. Physical Model and Assumptions. A single-stage planetary gear train (PGT) considered in this study consists of a sun gear and a ring gear which are coupled to each other by $N_{p}$ planets. Figure 2 illustrates a PGT with four planets, $N_{p}=4$, in which the ring gear and carrier are the input and output, respectively, while the sun gear is stationary. The ring gear load includes the vertical $F_{y}$ and axial forces $F_{z}$ in addition to the moment $T_{r}=R_{r} F_{x}$, where $R_{r}$ is base circle radius of the ring gear and $F_{x}$ is the circumferential force. The planets are mounted on a carrier through bearings and pins, and the carrier is supported flexibly in all directions. The dynamic model of the system shown in Figure 2 employs the following assumptions: 


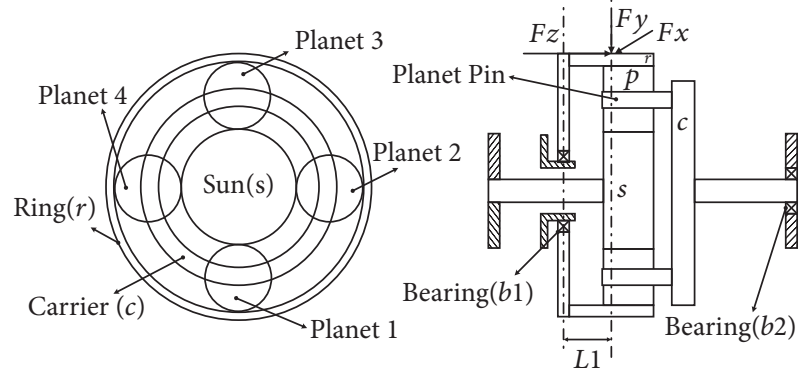

FIGURE 2: A typical PGT with four equally spaced planet gears under combined loading.

(i) The gear contact is treated as a solid-solid contact problem, that is, the elastohydrodynamic lubrication problem is not included in this study. The nonlinearity is due to the teeth separation, which will simplify the equations of motion.

(ii) The elastic deformations of gear bodies are assumed to be negligible.

(iii) The effects of the inertias connected to the PGT at the input and output sides are not included here considering that these can only cause resonances at low frequencies.

(iv) Frictional forces caused by tooth sliding motions are assumed to be negligible.

The coupled three-dimensional (transverse-rotationalaxial) model must be general so that a PGT with any number of planets can be analyzed, in which the planets can be spaced equally or unequally around the sun gear at any phasing relationship, and the sun and ring gear and carrier can be floating in the radial direction.

For a single-stage planetary gear system with $N_{p}$ planets, each planet is in mesh with the sun gear $(s)$ and the ring gear
( $r$ ) while it is supported through its axis by a bearing held by the carrier $(c)$. So any planet branch located at an arbitrary planet position angle $\psi_{k}$ can be considered as a combination of three subsystems: a sun and planet gear pair, a ring and planet gear pair and a carrier and planet gear pair, shown in Figure 3.

The dynamic model of a PGT allows each element including gears and the carrier to translate in $x, y$, and $z$ (axial) directions, denoted by $x_{j}, y_{j}$, and $z_{j}$, and rotate about these translation axes, denoted by $\theta_{x j}, \theta_{y j}$, and $\theta_{z j}(j=$ $\left.s, r, c, p_{1}, \ldots, p_{N p}\right)$. For the sake of convenience, define three variables in place of the rotations with $w_{x j}=R_{j} \theta_{x j}, w_{y j}=$ $R_{j} \theta_{y j}$, and $w_{z j}=R_{j} \theta_{z j}$, where $R_{j}$ is the base circle radius of a gear or the radius of the circle passing through planet centers of the carrier. Gears are modeled as rigid disks with masses $m_{j}$ and mass moments of inertias $I_{j}$. So a mass matrix $\mathbf{m}_{j}$ and a displacement vector $\mathbf{q}_{j}$ are then defined for each element $j$ as

$$
\begin{aligned}
\mathbf{m}_{j} & =\operatorname{diag}\left[\begin{array}{llllll}
m_{j} & m_{j} & m_{j} & \frac{I_{j}}{R_{j}^{2}} & \frac{I_{j}}{R_{j}^{2}} & \frac{I_{j}}{R_{j}^{2}}
\end{array}\right], \\
\mathbf{q}_{j} & =\left\{\begin{array}{lllllll}
x_{j} & y_{j} & z_{j} & w_{x j} & w_{y j} & w_{z j}
\end{array}\right\}^{T} .
\end{aligned}
$$

Before obtaining the dynamic model of the entire gear system, the equations of motion of these three subsystems will be formulated first based on lumped parameter method. Figure 3(a) illustrates an external helical gear pair, that is, the sun gear $s$ meshing with the planet gear- $k$ located at an arbitrary angle $\psi_{k}$. Figure 3(b) illustrates an internal helical gear pair, that is, the ring gear $r$ meshing with the same planet gear- $k$. Figure 3(c) illustrates the model of the carrier $c$ and the same planet gear- $k$ pair.

2.2. Model Formulation. The equations of motion of a single central gear- $i(i=r, s)$ and planet gear- $k\left(k=1, \ldots, N_{p}\right)$ pair are given as

$$
\begin{aligned}
& {\left[\begin{array}{cc}
\mathbf{m}_{i} & \mathbf{0} \\
\mathbf{0} & \mathbf{m}_{i}
\end{array}\right]\left\{\begin{array}{c}
\ddot{\mathbf{q}}_{i} \\
\ddot{\mathbf{q}}_{p k}
\end{array}\right\}+h_{i k} k_{i k}\left[\begin{array}{cc}
\mathbf{P}_{i k}^{T} \mathbf{P}_{i k} & -\mathbf{P}_{i k}^{T} \mathbf{P}_{i k} \\
-\mathbf{P}_{i k}^{T} \mathbf{P}_{i k} & \mathbf{P}_{i k}^{T} \mathbf{P}_{i k}
\end{array}\right]\left\{\begin{array}{c}
\mathbf{q}_{i} \\
\mathbf{q}_{p k}
\end{array}\right\}=\left\{\begin{array}{c}
\mathbf{f}_{i} \\
\mathbf{f}_{i p_{k}}
\end{array}\right\},} \\
& \mathbf{P}_{s k}=\left\{\cos \beta \cos \varphi_{s k}-\cos \beta \sin \varphi_{s k}-\sin \beta \sin \beta \cos \varphi_{s k}-\sin \beta \sin \varphi_{s k} \cos \beta\right\}, \\
& \mathbf{P}_{r k}=\left\{\cos \beta \cos \varphi_{r k}-\cos \beta \sin \varphi_{r k} \sin \beta \sin \beta \cos \varphi_{r k}-\sin \beta \sin \varphi_{r k}-\cos \beta\right\}, \\
& \mathbf{f}_{s}=\left\{\begin{array}{c}
0 \\
0 \\
0 \\
0 \\
\frac{T_{s}}{\left(N_{p} R_{s}\right)}
\end{array}\right\}+h_{s k} k_{s k} e_{s k}\left\{\begin{array}{c}
\cos \beta \cos \varphi_{s k} \beta \sin \varphi_{s k} \\
-\sin \beta \\
-\sin \beta \cos \varphi_{s k} \\
\sin \beta \sin \varphi_{s k} \\
-\cos \beta
\end{array}\right\},
\end{aligned}
$$




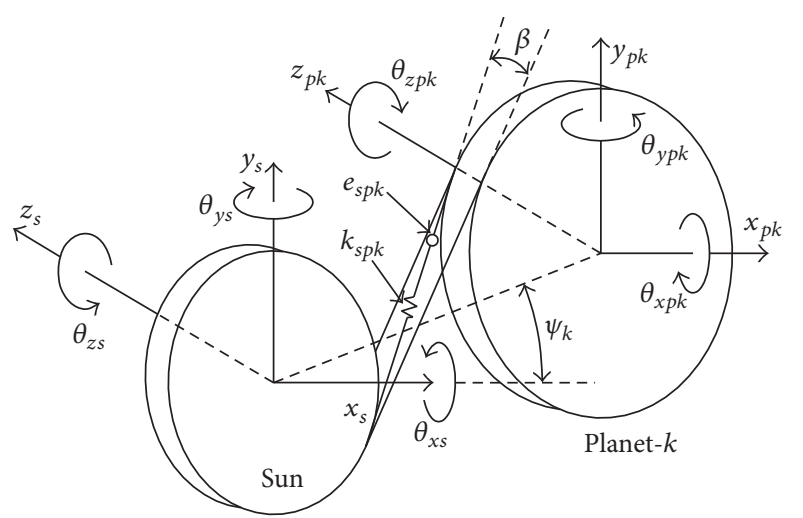

(a)

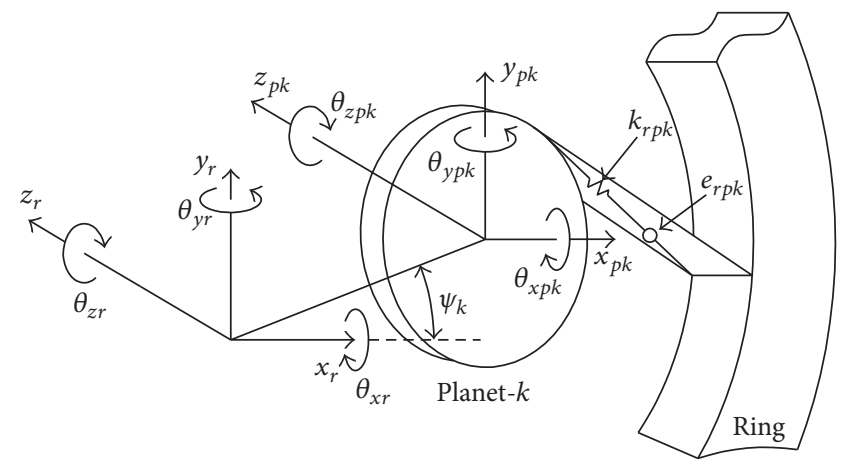

(b)

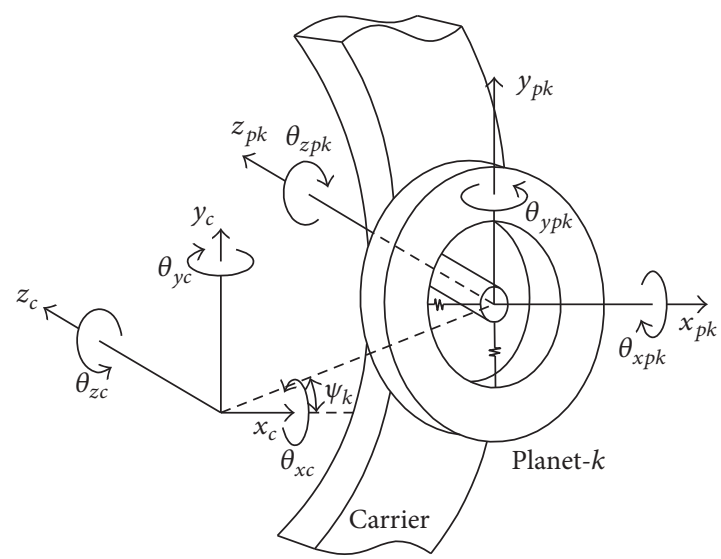

(c)

FIGURE 3: Dynamic models of (a) a sun and planet gear pair, (b) a ring and planet gear pair, and (c) a carrier and planet gear pair.

$$
\begin{aligned}
& \mathbf{f}_{s p_{k}}=h_{s k} k_{s k} e_{s k}\left\{\begin{array}{c}
-\cos \beta \cos \varphi_{s k} \\
\cos \beta \sin \varphi_{s k} \\
\sin \beta \\
-\sin \beta \cos \varphi_{s k} \\
\sin \beta \sin \varphi_{s k} \\
-\cos \beta
\end{array}\right\}, \\
& \left.\mathbf{f}_{r}=\left\{\begin{array}{c}
0 \\
0 \\
0 \\
0 \\
0 \\
\frac{T_{r}}{\left(N_{p} R_{r}\right)}
\end{array}\right\} \begin{array}{c}
\cos \beta \cos \varphi_{r k} \\
-\cos \beta \sin \varphi_{r k} \\
\sin \beta \\
\sin \beta \cos \varphi_{r k} \\
-\sin \beta \sin \varphi_{r k} \\
-\cos \beta
\end{array}\right\}, \\
& \mathbf{f}_{r p_{k}}=-\mathbf{f}_{r},
\end{aligned}
$$

where $\beta$ is the helical angle; $T_{i}$ is the mean torque applied to gear $-i ; \varphi_{i k}=\psi_{k}-\delta_{i k} \alpha_{i}$, where $\alpha_{i}$ is the transverse operating pressure angle and $\delta_{i k}=1$ for external gear pair and $\delta_{i k}=-1$ for internal gear pair; $k_{i k}$ is the meshing stiffness of gear- $i$ and 
planet gear- $k ; h_{i k}=0$ if the teeth lose contact and otherwise $h_{i k}=1$ according to the assumption of solid-solid contact; $e_{i k}$ is the static transmission error (TE) excitation function. And the relative gear mesh displacement along the line of action $p_{i k}$ is given as

$$
p_{i k}=\left[\begin{array}{ll}
\mathbf{P}_{i k} & -\mathbf{P}_{i k}
\end{array}\right]\left[\begin{array}{c}
\mathbf{q}_{i} \\
\mathbf{q}_{p_{k}}
\end{array}\right]-e_{i k} .
$$

The planet is attached to the carrier through a pin and a bearing. Since the planet gear is free to rotate, the torsional motion of the planet gear is uncoupled from the carrier motions. The relative displacement along all axis, denoted by $p_{x c k}, p_{y c k}$, and $p_{z c k}$, and rotation around these axis, denoted by $p_{\theta x c k}$ and $p_{\theta y c k}$, are given as

$$
\begin{aligned}
p_{x c k} & =x_{c}-x_{k}+w_{z c} \sin \psi_{k}, \\
p_{y c k} & =y_{c}-y_{k}-w_{z c} \cos \psi_{k}, \\
p_{z c k} & =z_{c}-z_{k}+w_{y c} \cos \psi_{k}-w_{x c} \sin \psi_{k}, \\
p_{\theta x c k} & =\theta_{x c}-\theta_{x k}, \\
p_{\theta y c k} & =\theta_{y c}-\theta_{y k} .
\end{aligned}
$$

The pin-bearing assembly is represented by the stiffness $k_{x}, k_{y}, k_{z}, k_{\theta x}$, and $k_{\theta y}$. So the equations of motion of the carrier $c$ and planet gear- $k$ pair are given as

$$
\begin{aligned}
& {\left[\begin{array}{cc}
\mathbf{m}_{c} & \mathbf{0} \\
\mathbf{0} & \mathbf{m}_{p_{k}}
\end{array}\right]\left\{\begin{array}{c}
\ddot{\mathbf{q}}_{c} \\
\ddot{\mathbf{q}}_{p k}
\end{array}\right\}+k_{i k}\left[\begin{array}{cc}
\mathbf{K}_{c 11 k} & -\mathbf{K}_{c 12 k} \\
-\mathbf{K}_{c 12 k}^{T} & \mathbf{K}_{c 22 k}
\end{array}\right]\left\{\begin{array}{c}
\mathbf{q}_{c} \\
\mathbf{q}_{p k}
\end{array}\right\}=\left\{\begin{array}{c}
\mathbf{f}_{c} \\
\mathbf{0}
\end{array}\right\},} \\
& \mathbf{K}_{c 11 k}=\left[\begin{array}{cccccc}
k_{y} & 0 & 0 & 0 & 0 & -k_{y} \cos \psi_{k} \\
0 & k_{x} & 0 & 0 & 0 & k_{x} \sin \psi_{k} \\
0 & 0 & k_{z} & k_{z} \cos \psi_{k} & -k_{z} \sin \psi_{k} & 0 \\
0 & 0 & k_{z} \cos \psi_{k} \frac{k_{\theta y}}{R_{c}^{2}}+k_{z} \cos ^{2} \psi_{k} & -k_{z} \cos \psi_{k} \sin \psi_{k} & 0 \\
0 & 0 & -k_{z} \sin \psi_{k} & -k_{z} \cos \psi_{k} \sin \psi_{k} & \frac{k_{\theta x}}{R_{c}^{2}}+k_{z} \sin ^{2} \psi_{k} & 0 \\
-k_{y} \cos \psi_{k} & k_{x} \sin \psi_{k} & 0 & 0 & 0 & k_{x} \sin ^{2} \psi_{k}+k_{y} \cos ^{2} \psi_{k}
\end{array}\right] \text {, } \\
& \mathbf{K}_{c 12 k}=\left[\begin{array}{cccccc}
-k_{y} & 0 & 0 & 0 & 0 & 0 \\
0 & -k_{x} & 0 & 0 & 0 & 0 \\
0 & 0 & -k_{z} & 0 & 0 & 0 \\
0 & 0 & -k_{z} \cos \psi_{k} & \frac{-k_{\theta y} R_{c}}{r_{k}} & 0 & 0 \\
0 & 0 & k_{z} \sin \psi_{k} & 0 & \frac{-k_{\theta x} R_{c}}{r_{k}} & 0 \\
k_{y} \cos \psi_{k} & -k_{x} \sin \psi_{k} & 0 & 0 & 0 & 0
\end{array}\right] \text {, } \\
& \mathbf{f}_{r}=\left\{\begin{array}{c}
0 \\
0 \\
0 \\
0 \\
0 \\
\frac{T_{c}}{\left(N_{p} R_{c}\right)}
\end{array}\right\} \\
& \mathbf{K}_{c 22 k}=\operatorname{diag}\left[\begin{array}{llllll}
k_{y} & k_{x} & k_{z} & \frac{k_{\theta y}}{R_{k}^{2}} & \frac{k_{\theta x}}{R_{k}^{2}} & 0
\end{array}\right] .
\end{aligned}
$$

In addition to the gear pairs, the elastic shafts and bearings also influence the dynamic characteristics of the gear transmission. Here, the housing holding some bearings is assumed rigid. Considering that the system has $N_{b}$ bearings, the overall bearing stiffness matrix $\mathbf{K}_{b}$ can be written in terms of individual bearing stiffness $\mathbf{K}_{b l}\left(l=1,2, \ldots, N_{b}\right)$ as $\mathbf{K}_{b}=\operatorname{diag}\left[\begin{array}{llll}\mathbf{K}_{b 1} & \mathbf{K}_{b 2} & \cdots & \mathbf{K}_{b N b}\end{array}\right]$. The model of shafts is developed with the finite element method (FEM). The stiffness 
and mass matrices of each finite shaft elements $m(m=$ $\left.1,2, \ldots, m_{s n}\right)$ of each individual shaft $n\left(n=1,2, \ldots, N_{s}\right)$ can be calculated to be a stiffness $\mathbf{K}_{s n}$ and mass $\mathbf{M}_{s n}$ matrices of $6 v_{n}\left(v_{n}=m_{s n}+1\right)$ dimension. Assembling all shaft stiffness $\mathbf{K}_{s}$ and mass matrices $\mathbf{M}_{s}$ of the system of $6 \sum_{1}^{N_{s}} v_{n}$ dimensions:

$$
\begin{aligned}
& \mathbf{K}_{s}=\operatorname{diag}\left[\begin{array}{llll}
\mathbf{K}_{s 1} & \mathbf{K}_{s 2} & \cdots & \mathbf{K}_{s N_{s}}
\end{array}\right], \\
& \mathbf{M}_{s}=\operatorname{diag}\left[\begin{array}{llll}
\mathbf{M}_{s 1} & \mathbf{M}_{s 2} & \cdots & \mathbf{M}_{s N_{s}}
\end{array}\right] .
\end{aligned}
$$

As a gear pair connects one node on one shaft to the other node on the next shaft according to (2a) and (5a), overall gear mass and stiffness matrices can be assembled with those of shafts and bearings. So the mass and stiffness matrices of the system are given as $\mathbf{M}=\mathbf{M}_{g}+\mathbf{M}_{s}$ and $\mathbf{K}=\mathbf{K}_{g}+\mathbf{K}_{s}+\mathbf{K}_{b}$.

The damping matrix $\mathbf{C}$ is not derived to this point in the formulation. That is because the damping values are not known in most cases even in the final stages of gear design. Therefore, for practical engineering purpose, a set of constant damping values are used in the forces response calculations based on the experience on damping of gear meshes. Here, 2.5 percent modal damping is used when solving the forced response.

Based on above equations, the three-dimensional dynamic model of the overall single-state planetary gear system with $N_{p}$ planets can be written in matrix form as

$$
\mathbf{M} \ddot{\mathbf{X}}(t)+\mathbf{C} \dot{\mathbf{X}}(t)+\mathbf{K X}(t)=\mathbf{F} .
$$

2.3. Gear Contact Model. In order to analyze the forced vibrations with above dynamic models, the excitations such as the gear transmission error and stiffness and manufacturing errors should be defined up-front through the loaded gear teeth contact analysis. A load distribution model considering the conditions of compatibility and equilibrium was initially proposed by Conry and Serieg [11] based on Hertz mechanics and developed by the researchers from the gear and powertrain lab of Ohio State University [12]. Given the tooth compliance, applied torque, and the initial tooth separation, elastic deformations at any point of the gear surface are computed by using a modified Simplex algorithm. Yue et al. [13] analyzed the teeth contact characteristics of helical gear pairs with a finite element model and proposed a computational method based on genetic algorithm to optimize gear profile parameters to reduce the transmission error, which is used here to calculate the excitations, whose detailed modeling and simulation was introduced in [14] and not discussed in this study.

The mesh stiffness due to tooth bending, base rotation, and shear deformations is computed by using simplified finite element formulations as a function of gear rotation. The time-varying transmission error and mesh stiffness of each external and internal gear pair are predicted as a periodic function at the mesh frequency of the gear mesh, written as

$$
e_{s k}(t)=\sum_{l=1}^{L} e_{s k}^{(l)} \sin \left(l \omega_{m} t+l Z_{s} \psi_{k}+\phi_{s k}^{(l)}\right)
$$

$$
\begin{aligned}
& e_{r k}(t)=\sum_{l=1}^{L} e_{r k}^{(l)} \sin \left(l \omega_{m} t+l Z_{r} \psi_{k}+l \psi_{s r}+\phi_{r k}^{(l)}\right), \\
& k_{s k}(t)=\bar{k}_{s k}+\sum_{l=1}^{L} k_{s k}^{(l)} \sin \left(l \omega_{m} t+l Z_{s} \psi_{k}+\phi_{s k}^{(l)}+l \gamma_{t s}\right), \\
& k_{r k}(t) \\
& \quad \bar{k}_{r k} \\
& \quad+\sum_{l=1}^{L} k_{r k}^{(l)} \sin \left(l \omega_{m} t+l Z_{r} \psi_{k}+l \gamma_{s r}+\phi_{r k}^{(l)}+l \gamma_{t s}\right) .
\end{aligned}
$$

Here, $e_{i k}^{(l)}$ and $\phi_{i k}^{(l)}(i=s, r)$ are the amplitude and phase angle of the $l$ th harmonic term; $\bar{k}_{i k}$ and $k_{i k}^{(l)}(i=s, r)$ are the average and alternating mesh stiffness amplitude; the gear mesh frequency $\omega_{m}$ is defined as $\omega_{m}=Z_{s}\left|\omega_{m}-\omega_{c}\right|=$ $Z_{r}\left|\omega_{r}-\omega_{c}\right|$, where $\omega_{i}$ and $Z_{i}$ are absolute angular velocity and teeth number of gear $i$ and $\omega_{c}$ is the absolute angular velocity of the carrier; $L$ is the total number of harmonic term considered; $\gamma_{s r}$ is the phase angle between $e_{s k}(t)$ and $e_{r k}(t) ; \gamma_{t s}$ is the phase angle between time-varying transmission error and mesh stiffness.

\section{An Example Analysis}

A 4-planet planetary gear set in Figure 2 is used here as an application example, where the sun is stationary; the ring gear and the carrier are the input and output, respectively. The external vertical and axial loads in addition to the moment transmitted from a counter-shaft gear pair are imposed on the ring gear, and a design parameter $L 1$ is defined by the distance between the middle plane of the counter-shaft gear pair and the middle plane of the ring bearing, $L 1(0 \leq L 1 \leq L 0)$ where the maximum $L 0$ is determined by the widths of the gears and the bearing. One part of the imposed load is finally burdened by the bearing of the ring gear, and the other part is burdened by the ring gear and finally transferred to the sun gear through planet gears. The load shares depend on the value of $L 1$ according to Newton's equilibrium law; that is, there is the minimum load share on the ring gear at $L 1=0$ while the maximum is at $L 1=L 0$.

Table 1 lists the basic gear parameters of the example gear train. The planets are equally spaced but not in phase [15] since $Z_{i} / Z_{p} \neq$ integer for equally spaced planets. Meanwhile, the dynamic mesh forces are sequentially phased; that is, sum of the phase angles is an integer multiple of $\pi$. This case is one of the most common conditions used in product applications because of its lower vibration and noise levels [16]. Table 2 lists the input moment and forces of the ring gear for gear contact model, which corresponds to about $80 \%$ of the related maximum moment of this planetary gear set in an actual automatic transmission. To be convenient for comparison, the traditional driving way is named as "pure moment loading" while the driving way in this study is named as "combined loading" since the driving source includes not only the moment but also the vertical and axial forces. 


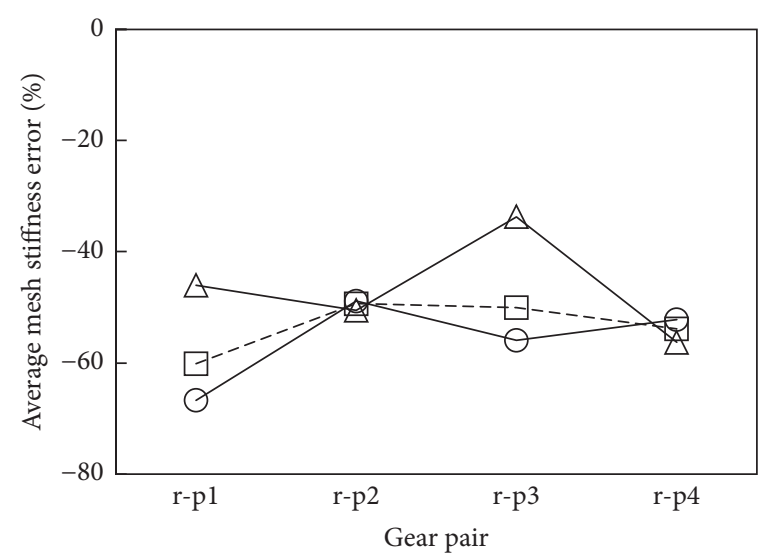

$$
\begin{aligned}
& \triangle L 1=0 \\
& \square L 1=L 0 / 2 \\
& \bigcirc L 1=L 0
\end{aligned}
$$

(a)

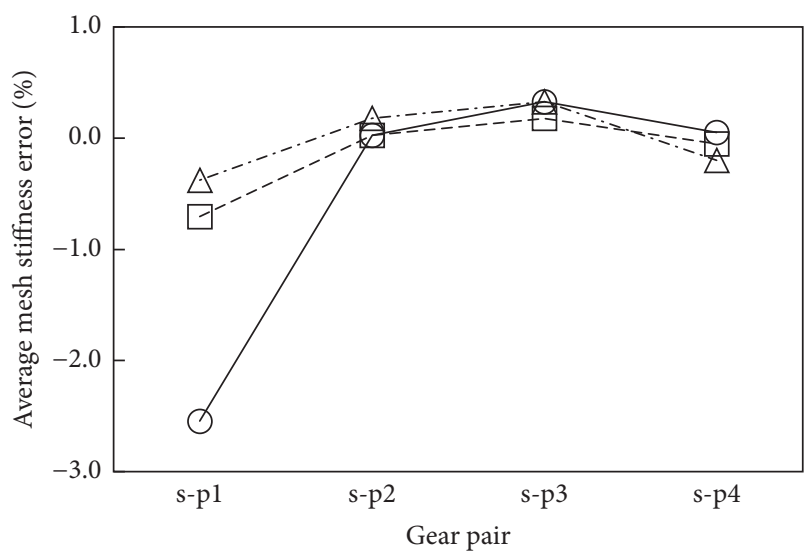

$$
\begin{aligned}
& \triangle-L 1=0 \\
& -\square 1=L 0 / 2 \\
& -L 1=L 0
\end{aligned}
$$

FIGURE 4: The influence of the design parameter $L 1$ on average mesh stiffness (a) ring-planet gear pairs and (b) sun-planet gear pairs.

TABLE 1: Basic design parameters of the example gear train.

\begin{tabular}{lccc}
\hline Parameter & Sun & Ring & Planet \\
\hline Number of teeth & 23 & 73 & 24 \\
Module $(\mathrm{mm})$ & 1.47 & & \\
Pressure angle $\left(^{\circ}\right)$ & 17.5 & & \\
Helical angle $\left(^{\circ}\right)$ & 15.5 & & \\
Face width $(\mathrm{mm})$ & 18 & 20 & 19 \\
Root diameter $(\mathrm{mm})$ & 31.25 & 114.61 & 32.45 \\
Outside diameter $(\mathrm{mm})$ & 39.59 & & 40.79 \\
Minor diameter $(\mathrm{mm})$ & - & 106.81 & - \\
Tooth thickness $(\mathrm{mm})$ & 2.58 & 2.67 & 2.48 \\
Backlash $(\mu \mathrm{m})$ & 74 & & \\
\hline
\end{tabular}

TABLE 2: The imposed combined forces and moment loading on the ring gear.

\begin{tabular}{lccc}
\hline$F_{x}(\mathrm{~N})$ & $F_{y}(\mathrm{~N})$ & $F_{z}(\mathrm{~N})$ & $T_{r}=R F_{x}(\mathrm{Nm})$ \\
\hline 4267 & 1405 & 1387 & 385 \\
\hline
\end{tabular}

Table 3 shows the results of the power flow analysis. It is observed from these results that imposed vertical and axial forces will lead to obvious disparity of mesh forces and stiffness between planets, that is, the uniform load sharing between planets is deteriorated. With the condition of combined loading, the additional imposed vertical and axial forces at different position on the ring gear would change average gear mesh stiffness while they would not change the gear mesh forces since there is different elastic shaft deflection at different position even with the same vertical and axial forces. Figure 4 shows the relative deviation of calculated average mesh stiffness under the condition of combined loading by comparing with the pure moment loading condition, which has the following features:

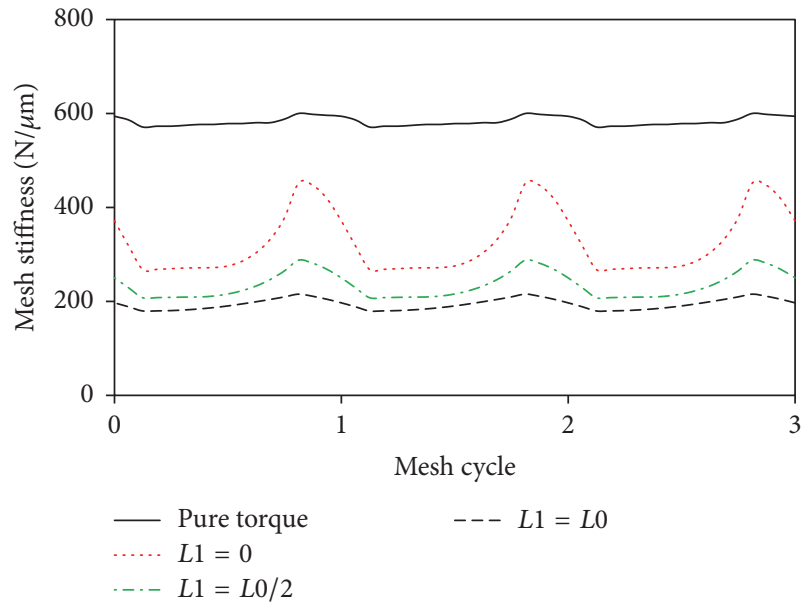

FIGURE 5: Time-varying mesh stiffness of ring-planet 1 under different loading conditions.

(i) The average mesh stiffness of each gear pair is different when imposing the additional vertical and axial forces, not similar to those under the condition of pure torque loading. The maximum change is for gear pairs with planet 1 whose radial position is collinear to the additional vertical force direction and away from the imposed force position.

(ii) The average mesh stiffness of ring-planet gear pairs is reduced seriously when imposing additional vertical and axial forces on the ring gear. The maximum reduction is for the planet 1 at $L 1=L 0$. The detailed time-varying mesh stiffness of ring-planet 1 is presented in Figure 5. The reduction of the average mesh stiffness is tremendous for ring-planet gear pairs and mild for sun-planet gear pairs. Here, the ring gear is the driving gear and so the kinetic transfer path from 
TABLE 3: Static gear mesh forces and average gear mesh stiffness values.

\begin{tabular}{|c|c|c|c|c|c|c|}
\hline \multicolumn{3}{|c|}{ Pure torque loading } & \multicolumn{4}{|c|}{ Combined loading } \\
\hline & \multirow{2}{*}{$\begin{array}{l}\text { Mesh force } \\
(\mathrm{N})\end{array}$} & \multirow{2}{*}{$\begin{array}{l}\text { Mesh stiffness } \\
\quad(\mathrm{N} / \mu \mathrm{m})\end{array}$} & \multirow{2}{*}{$\begin{array}{l}\text { Mesh force } \\
\quad(\mathrm{N})\end{array}$} & \multicolumn{3}{|c|}{ Mesh stiffness $(\mathrm{N} / \mu \mathrm{m})$} \\
\hline & & & & $L 1=0$ & $L 1=L 0 / 2$ & $L 1=L 0$ \\
\hline s-p1 & 1697.2 & 396.9 & 1505.5 & 395.4 & 394.1 & 386.8 \\
\hline s-p2 & 1699.9 & 396.9 & 1814.1 & 397.6 & 397.0 & 397.0 \\
\hline s-p3 & 1697.2 & 396.9 & 1882.4 & 398.2 & 397.6 & 398.2 \\
\hline s-p4 & 1699.9 & 396.9 & 1587.5 & 396.1 & 396.7 & 397.1 \\
\hline $\mathrm{r}-\mathrm{p} 1$ & 1769.5 & 581.4 & 1569.5 & 313.9 & 231.8 & 193.4 \\
\hline $\mathrm{r}-\mathrm{p} 2$ & 1772.3 & 581.4 & 1891.3 & 287.4 & 294.7 & 297.3 \\
\hline $\mathrm{r}-\mathrm{p} 3$ & 1769.5 & 581.4 & 1962.5 & 385.3 & 290.7 & 256.2 \\
\hline $\mathrm{r}-\mathrm{p} 4$ & 1772.3 & 581.4 & 1655.0 & 254.2 & 268.4 & 277.8 \\
\hline
\end{tabular}

Note. The symbol s-pl represents the sun-planet 1 gear pair and the like.

ring gear to sun gear through planet gears can weaken the changes of average mesh stiffness of sun-planet gear pairs.

(iii) With the increasing of the design parameter $L 1$, starting at 0 to $L 0$, the average mesh stiffness is reduced for all gear pairs.

Figure 6 shows an example of the load distribution of planet 3 with different design parameter $L 1$. It is obvious that there is less distributed load on planet 3 when there is minimal load share on the ring gear; that is, $L 1=0$. Meanwhile, the bias load distribution on meshing gears is found. The imposed vertical load would lead to the bending of shafts of the planetary gear set and so destroy the uniformity of load distribution.

The undamped natural frequencies $\omega^{e}$ predicted by the model are listed in Table 4. It is observed that the elastic model modifies the original results $\omega^{\Re}$ of the rigid model. The maximum modification $\left(\omega^{e}-\omega^{\Re}\right) / \omega^{\Re}$ is about $\pm 10 \%$ for low orders such as below order 10th, and less than $-20 \%$ for high orders such as order 20th, shown in Figure 7(a). The relative error to the result at $L 1=0$, calculated as $\left(\omega^{e}-\omega_{L 1=0}^{e}\right) / \omega_{L 1=0}^{e}$, is shown in Figure $7(\mathrm{~b})$. It is observed that nonzero design parameter $L 1$ will reduce natural frequencies for low orders, such as before 16 orders here, due to the cantilever effect.

The amplitudes, $e_{s k}^{(l)}$ and $e_{r k}^{(l)}$, of the first five mesh harmonics $(l \in[1, L])$ of the static transmission error excitations are given in Table 5 for pure moment loading and combined loading conditions. Here, phase angles, $\phi_{s k}^{(l)}$ and $\phi_{r k}^{(l)}$ of each harmonic are assumed to be zero. The steady-state response $\mathbf{X}$ of the planetary gear set to the force vector $\mathbf{F}$ defined by (2a) is predicted. The dynamic force on each gear mesh is then obtained using the expression

$$
\begin{aligned}
F_{i k}(t)=c_{i k}(t) \dot{p}_{i k}(t)+h_{i k} k_{i k}(t) & p_{i k}(t) \\
& \left(i=s, r k=1, \ldots, N_{p}\right) .
\end{aligned}
$$

The proposed dynamic model is used to analyze the forced vibration responses at pure and combined loading conditions. Figures 8 and 9 show a set of curves of dynamic
TABle 4: Predicted natural frequencies of the example gear train system.

\begin{tabular}{lccc}
\hline \multirow{2}{*}{ Rigid shaft $\omega^{\Re}(\mathrm{Hz})$} & \multicolumn{3}{c}{ Elastic shaft $\omega^{e}(\mathrm{~Hz})$} \\
\hline 2.9 & 2.8 & $L 1=L 0 / 2$ & $L 1=L 0$ \\
468.8 & 447.6 & 472.2 & 2.9 \\
622.2 & 584.9 & 612.3 & 559.6 \\
715.5 & 695.5 & 789.2 & 772.9 \\
978.6 & 962.9 & 978.9 & 983.3 \\
1112.7 & 1071.7 & 1112.6 & 1112.7 \\
1168.2 & 1112.7 & 1128.3 & 1142.1 \\
1210.5 & 1138.2 & 1169.9 & 1206.9 \\
1463.3 & 1370.8 & 1449.9 & 1517.6 \\
1698.8 & 1698.5 & 1698.6 & 1698.6 \\
1976.5 & 1940.9 & 1959.7 & 1958.0 \\
2205.8 & 1991.6 & 2009.3 & 1996.5 \\
2496.3 & 2436.7 & 2511.2 & 2562.0 \\
3397.9 & 3193.5 & 3211.0 & 3220.5 \\
3566.6 & 3292.1 & 3305.9 & 3310.1 \\
4474.8 & 3865.4 & 3941.4 & 3938.1 \\
4517.4 & 4504.9 & 4504.5 & 4504.6 \\
4997.7 & 4611.5 & 4602.8 & 4672.5 \\
5539.0 & 4945.4 & 4885.9 & 4886.5 \\
6816.6 & 5233.9 & 5149.3 & 5156.6 \\
\hline
\end{tabular}

gear mesh forces for this system plotted against the mesh frequency. The following observations merit attention.

(i) For pure moment loading condition, the dynamic gear mesh forces are quite the same for each planet. It is noteworthy that the peak dynamic mesh force for sun-planet pair is smaller wholly than the one at pure torque loading condition, no matter what the design parameter $L 1$ is.

(ii) For combined loading condition, the dynamic gear mesh forces are different for each planet. There is a similar trend for planets on a diagonal line. The design parameter $L 1$ influences the dynamic gear mesh 


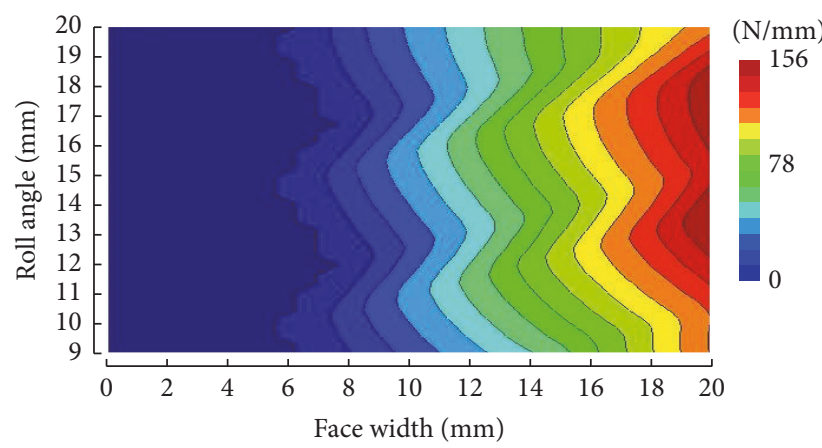

(a) Ring-planet 3 at $L 1=0$

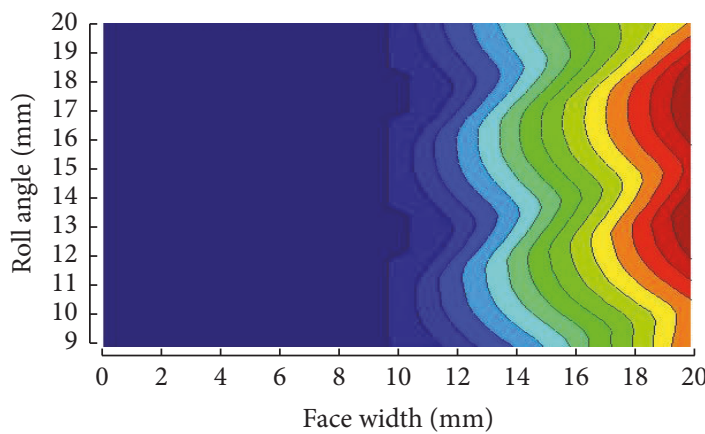

(c) Ring-planet 3 at $L 1=L 0 / 2$

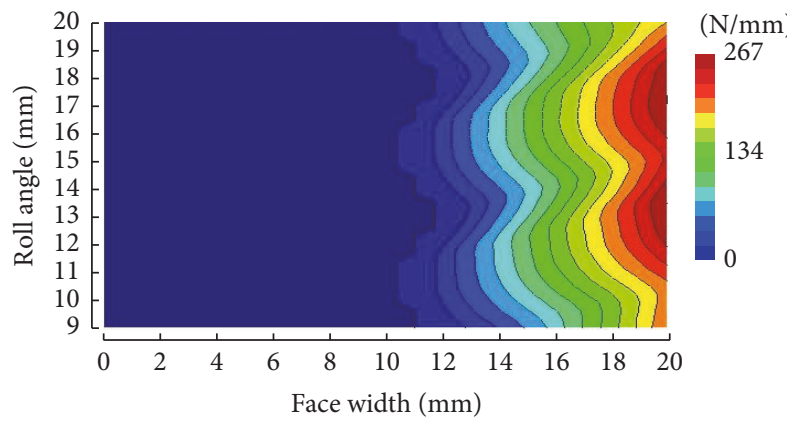

(e) Ring-planet 3 at $L 1=L 0$

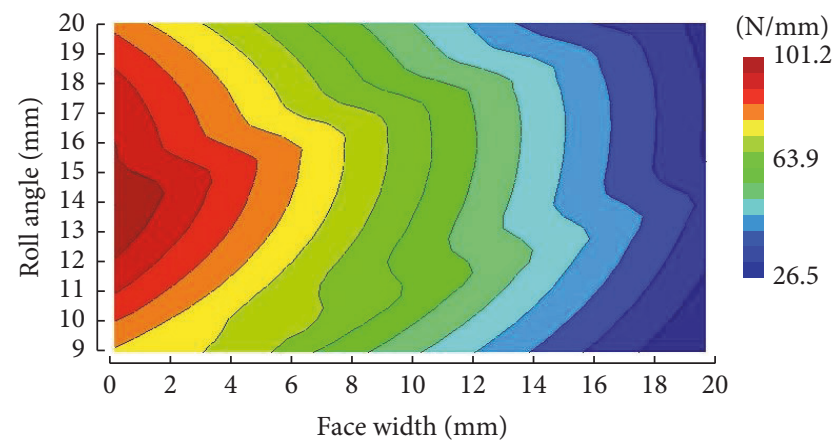

(b) Sun-planet 3 at $L 1=0$

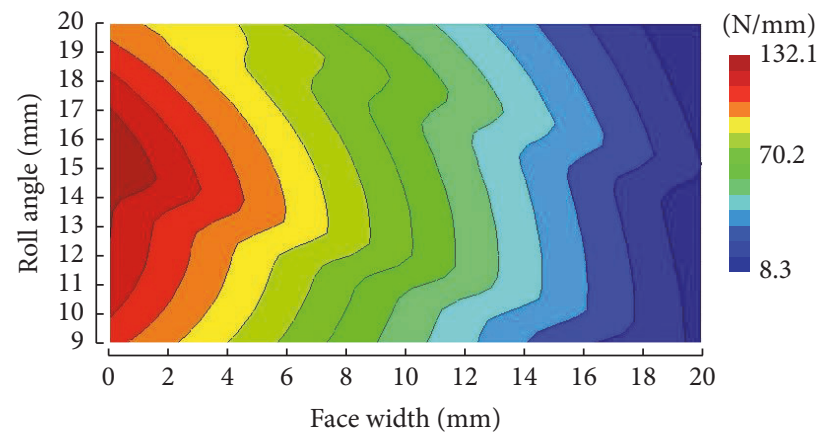

(d) Sun-planet 3 at $L 1=L 0 / 2$

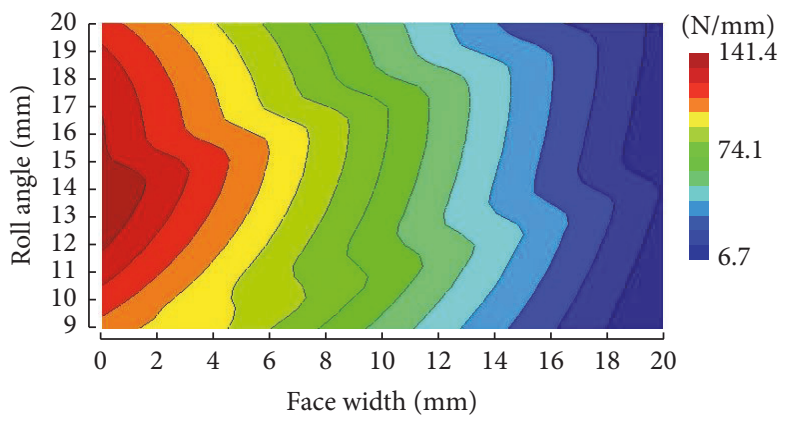

(f) Sun-planet 3 at $L 1=L 0$

Figure 6: Load distribution of planet 3 along the face width.

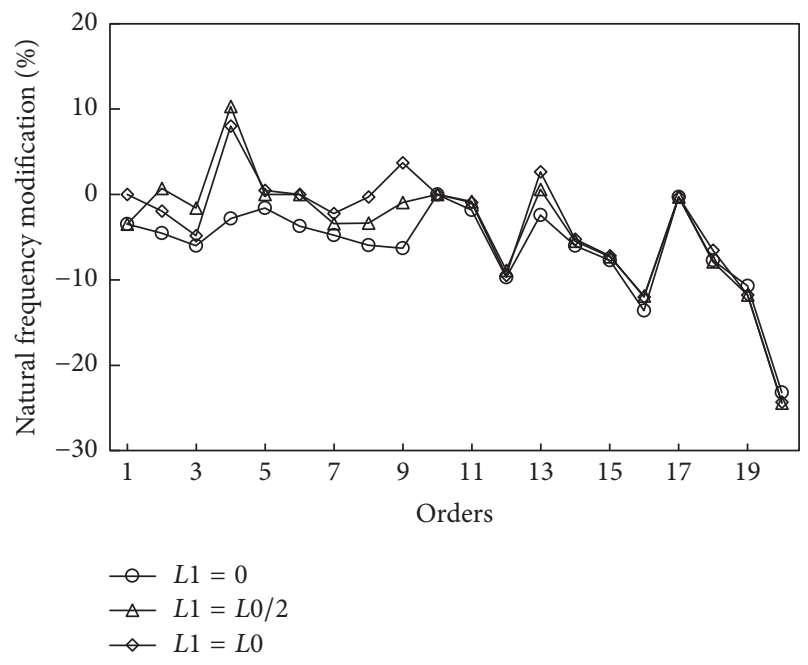

(a)

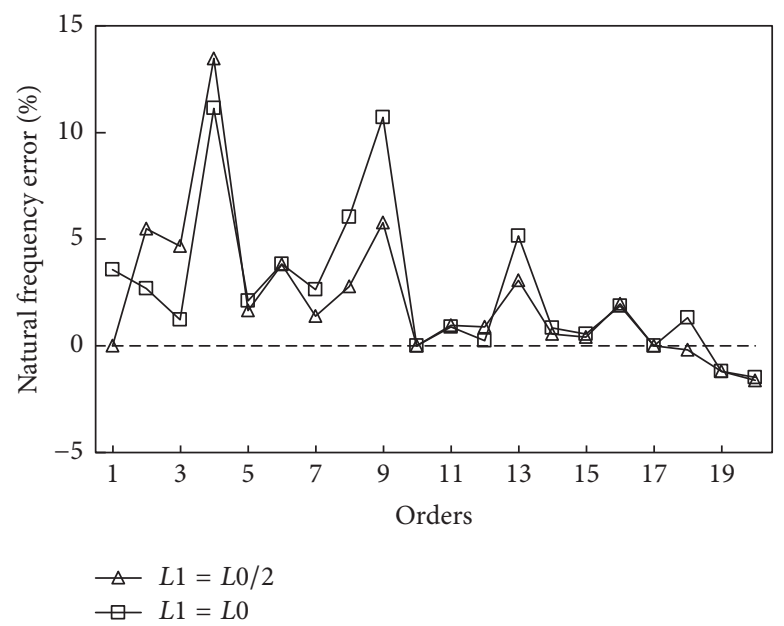

(b)

FIGURE 7: The predicted natural frequencies by the proposed dynamic model. (a) Modification to the results by rigid shaft model and (b) relative error to the results at $L 1=0$. 
TABLE 5: The first five harmonic amplitudes of the static transmission error excitations.

\begin{tabular}{|c|c|c|c|c|c|c|c|c|c|}
\hline & $l$ & s-p1 & s-p2 & s-p3 & s-p4 & r-p1 & r-p2 & r-p3 & r-p4 \\
\hline & 1 & \multicolumn{4}{|c|}{0.115} & \multicolumn{4}{|c|}{0.054} \\
\hline & 2 & \multicolumn{4}{|c|}{0.046} & \multicolumn{4}{|c|}{0.030} \\
\hline Pure torque loading & 3 & \multicolumn{4}{|c|}{0.022} & \multicolumn{4}{|c|}{0.004} \\
\hline & 4 & \multicolumn{4}{|c|}{0.008} & \multicolumn{4}{|c|}{0.009} \\
\hline & 5 & \multicolumn{4}{|c|}{-} & \multicolumn{4}{|c|}{-} \\
\hline \multirow{5}{*}{$L 1=0$} & 1 & 0.079 & 0.15 & 0.15 & 0.081 & 0.694 & 0.96 & 0.567 & 1.03 \\
\hline & 2 & 0.034 & 0.0595 & 0.0599 & 0.035 & 0.161 & 0.209 & 0.141 & 0.27 \\
\hline & 3 & 0.018 & 0.0287 & 0.029 & 0.018 & 0.023 & 0.026 & 0.015 & 0.043 \\
\hline & 4 & 0.0065 & 0.011 & 0.011 & 0.0068 & 0.035 & 0.034 & 0.022 & 0.059 \\
\hline & 5 & 0.0005 & 0.0016 & 0.0016 & 0.0005 & 0.036 & 0.032 & 0.019 & 0.057 \\
\hline \multirow{5}{*}{$L 1=L 0 / 2$} & 1 & 0.12 & 0.154 & 0.262 & 0.037 & 1.04 & 0.887 & 1.04 & 0.976 \\
\hline & 2 & 0.047 & 0.06 & 0.098 & 0.025 & 0.306 & 0.191 & 0.225 & 0.246 \\
\hline & 3 & 0.022 & 0.029 & 0.044 & 0.015 & 0.049 & 0.023 & 0.028 & 0.037 \\
\hline & 4 & 0.0077 & 0.011 & 0.016 & 0.0062 & 0.065 & 0.031 & 0.037 & 0.053 \\
\hline & 5 & 0.0005 & 0.0016 & 0.0023 & 0.0008 & 0.059 & 0.029 & 0.035 & 0.054 \\
\hline \multirow{5}{*}{$L 1=L 0$} & 1 & 0.214 & 0.104 & 0.283 & 0.046 & 1.21 & 0.851 & 1.33 & 0.954 \\
\hline & 2 & 0.078 & 0.044 & 0.105 & 0.028 & 0.426 & 0.182 & 0.323 & 0.234 \\
\hline & 3 & 0.034 & 0.022 & 0.048 & 0.016 & 0.066 & 0.022 & 0.044 & 0.035 \\
\hline & 4 & 0.012 & 0.009 & 0.018 & 0.006 & 0.074 & 0.03 & 0.058 & 0.049 \\
\hline & 5 & 0.0006 & 0.0013 & 0.0025 & 0.0007 & 0.081 & 0.028 & 0.045 & 0.053 \\
\hline
\end{tabular}
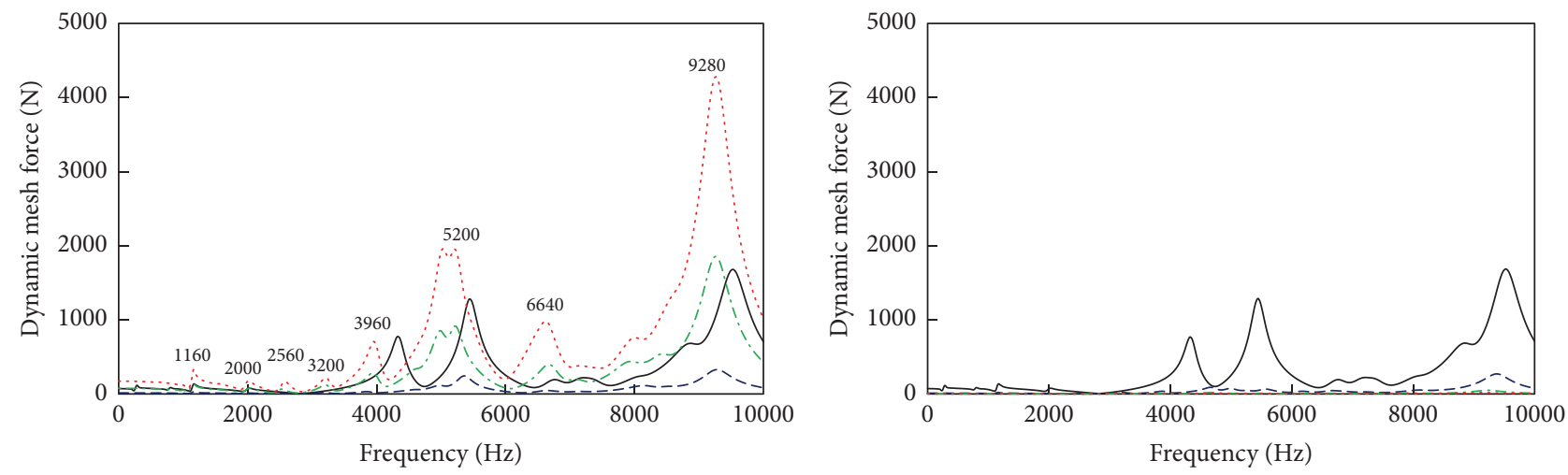

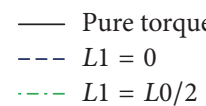

$\cdot-\cdot-L 1=L 0 / 2$

(a)

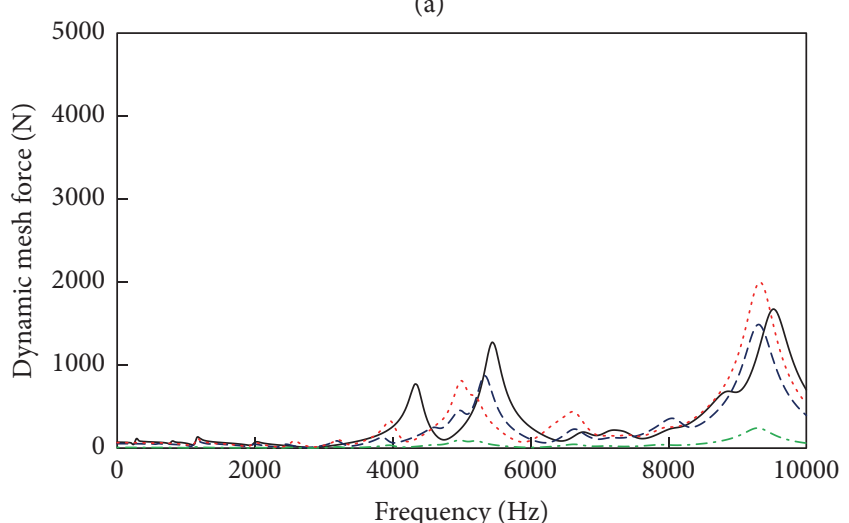

Pure torque
$---L 1=0$

$\cdots \cdot L 1=L 0$

-..- $L 1=L 0 / 2$

(c)
$---L 1=0$

-..- $L 1=L 0 / 2$

(b)

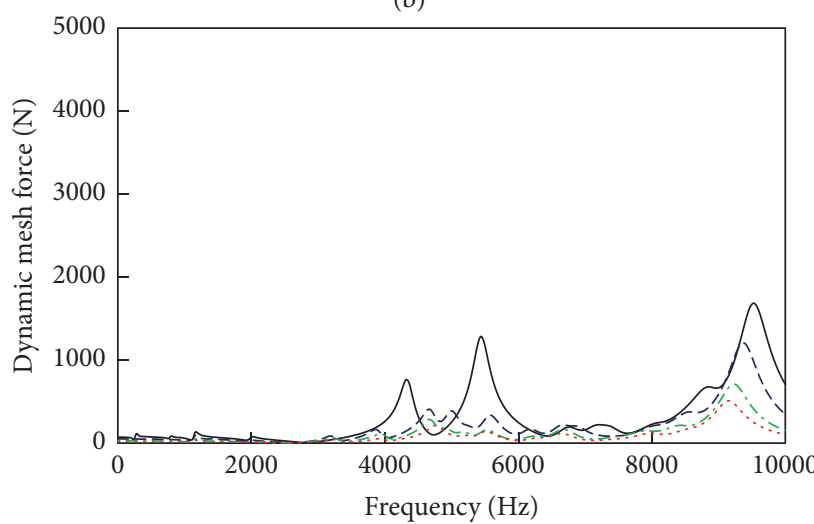

- Pure torque
-- $L 1=0$
-.- $L 1=L 0 / 2$ $\cdots \ldots . L 1=L 0$

(d)

FIGURE 8: Dynamic mesh forces at the ring-planet gear pairs with mixed dynamic model; (a) ring-planet 1, (b) ring-planet 2, (c) ring-planet 3 , and (d) ring-planet 4 . 

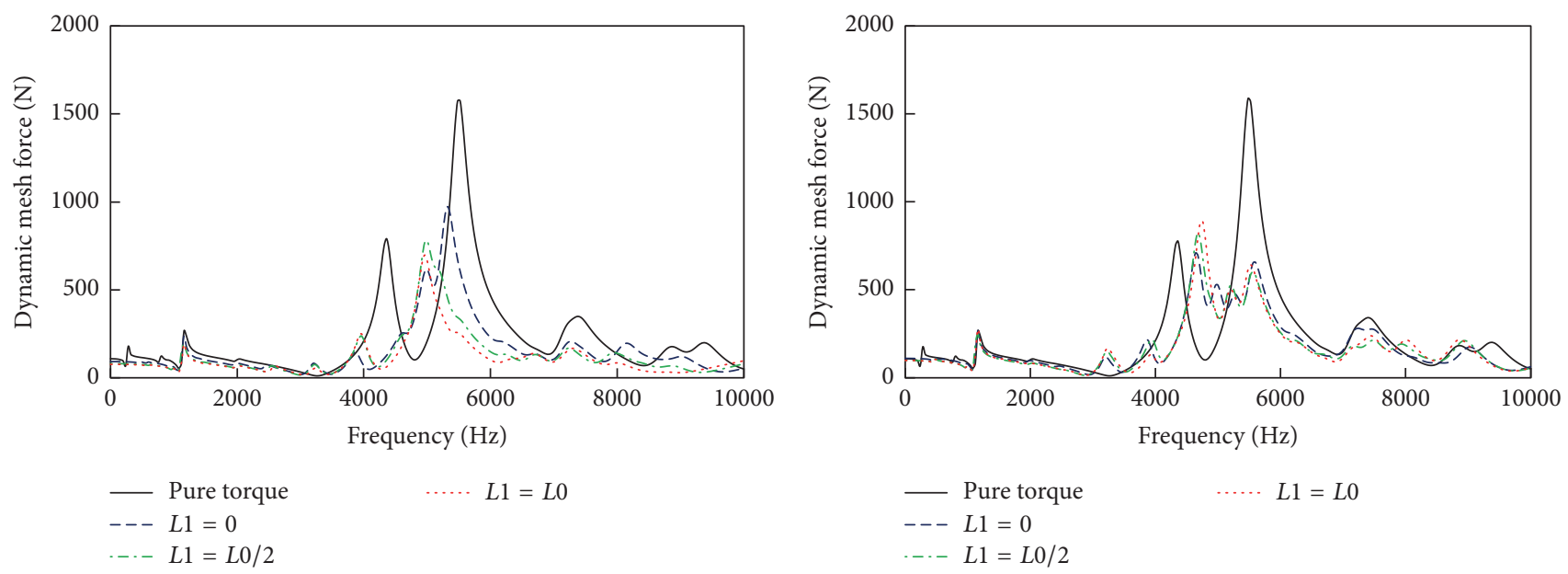

(a)

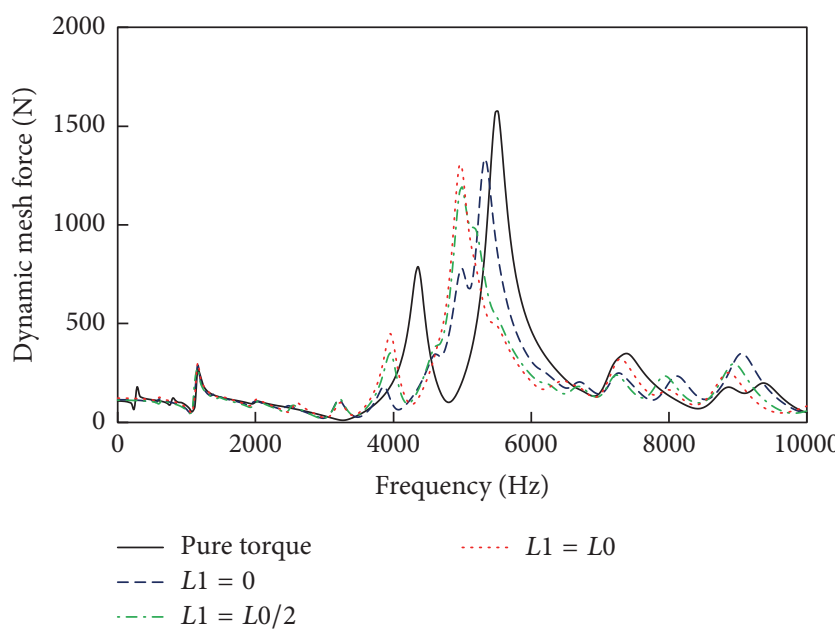

(c)

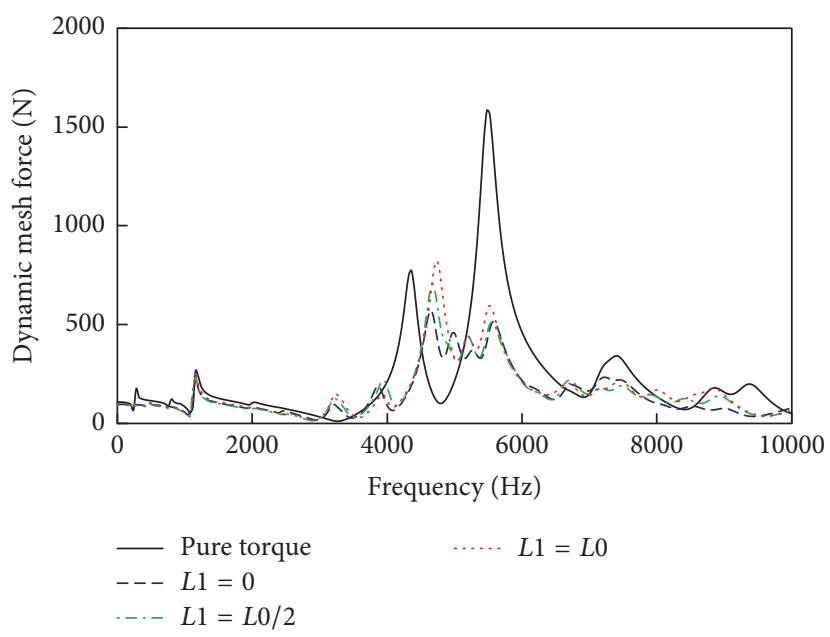

(d)

Figure 9: Dynamic mesh forces at the sun-planet gear pairs; (a) sun-planet 1, (b) sun-planet 2, (c) sun-planet 3, and (d) sun-planet 4.

forces of each ring-planet gear pair differently. The largest peak mesh force is for ring-planet 1 because of its special position. The increasing magnitude is about 2 times.

(iii) Peak dynamic mesh force amplitudes are observed at a number of frequencies. Each of these resonance peaks can be linked to a certain natural mode and certain transmission error harmonic amplitude exciting that particular mode. For instance, with the mixed dynamic model at $L 1=L 0$, the peak at $\omega_{m}=2560 \mathrm{~Hz}$ is due to the natural model at $\omega^{e}=2562.0 \mathrm{~Hz}$ excited by first harmonic of transmission errors, representing the condition $\omega_{m} \approx \omega^{e} / 1$, so as the other peaks.

Figure 10 shows a set of curves of dynamic bearing forces for this system plotted against the mesh frequency. The dynamic bearing force excited by the transmission error from sun-planet gear pairs or ring-planet gear pairs is almost identical and so only outstood in the sun-planet 1 and ringplanet 1, shown in Figures 10(a) and 10(b), by the right secondary axis because of different magnitudes. In the condition of pure moment loading, the reached bearing dynamic force originated from gear meshing is tiny. However, the magnitude of bearing forces under combine loading conditions is expanded significantly regardless of which gear tooth meshing the transmission error excitation is generated from. These dynamic forces will propagate from there to the vehicle body through the transmission mountings and thus lead to noise and vibration.

\section{Conclusions}

In this study, by combining the lumped parameter method with finite element method, a three-dimensional, nonlinear, time-variant dynamic model has been developed to simulate the dynamics of a single-stage helical planetary gear set under combined force and moment loading conditions. The periodic excitation functions have been derived in terms of functional transmission error and mesh stiffness parameters from an elastic contact analysis. The proposed model has been employed to investigate the effects of the combined loading and positioning on the overall dynamic behavior of a four-planet planetary gear set system quantitatively. 

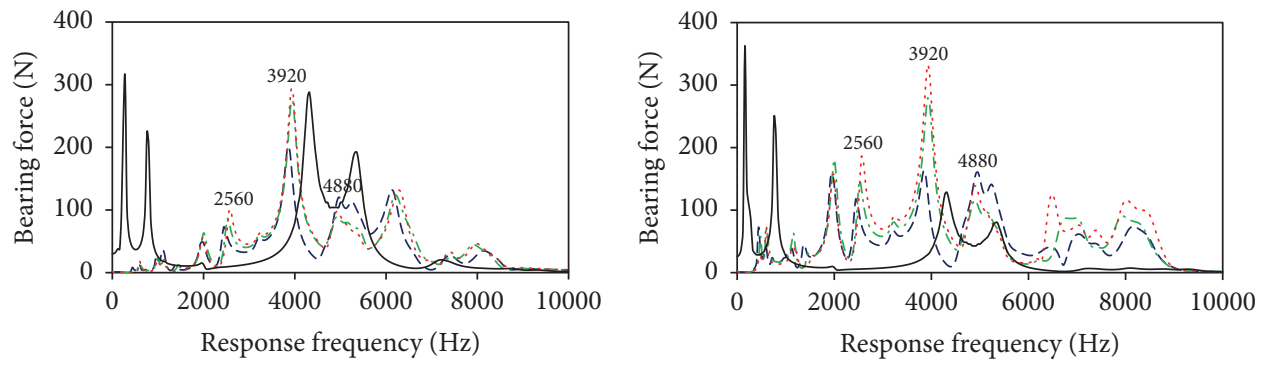

$$
\begin{aligned}
& --L 1=0 \quad-\text { Pure torque } \\
& -\cdot-L 1=L 0 / 2 \\
& \cdots \ldots 1=L 0
\end{aligned}
$$

(a)

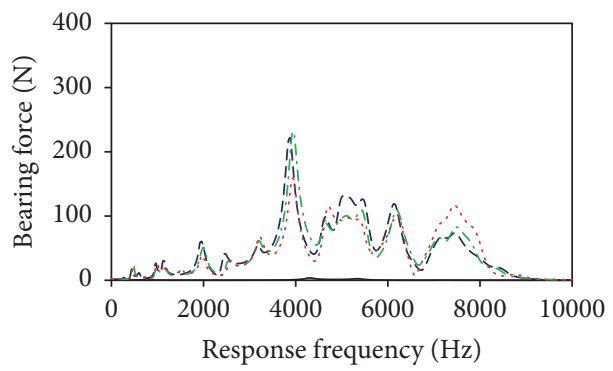

$$
\begin{aligned}
& \text { - Pure torque } \quad \cdots \cdots, L 1=L 0 \\
& ---L 1=0 \\
& --.-L 1=L 0 / 2
\end{aligned}
$$

(c)

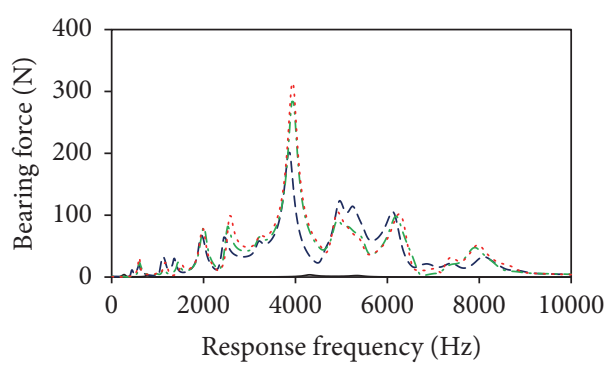

$$
\begin{array}{ll}
\text { - Pure torque } & \cdots \cdots \\
--- & L 1=0 \\
-- & L 1=L 0 / 2
\end{array}
$$

(e)

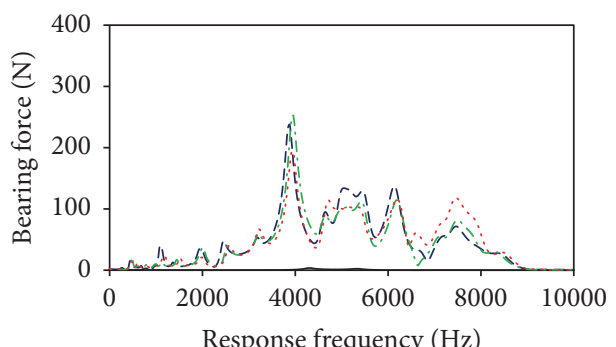

Response frequency $(\mathrm{Hz})$

$$
\begin{array}{ll}
\text { - Pure torque } & \cdots \cdots \\
--- & L 1=0 \\
-\cdots & L 1=L 0 / 2
\end{array}
$$

(g)

$$
\begin{aligned}
& ---L 1=0 \\
& -\cdot-L 1=L 0 / 2 \quad \text { Pure torque }
\end{aligned}
$$

(b)

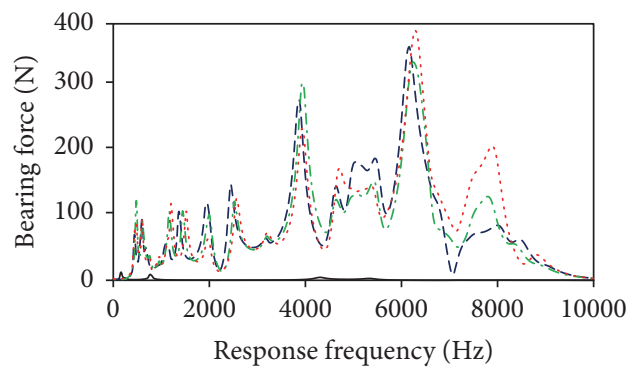

$$
\begin{aligned}
& \text { - Pure torque } \quad \cdots \cdots \cdot L 1=L 0 \\
& \text {-- } L 1=0 \\
& \text {-.. } L 1=L 0 / 2
\end{aligned}
$$

(d)

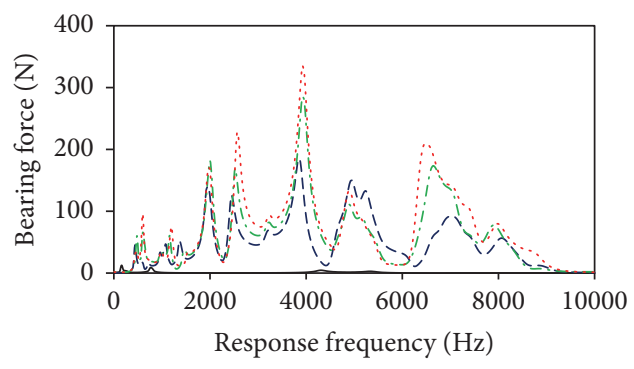

$$
\begin{array}{ll}
\text { - Pure torque } \cdots \cdots & L 1=L 0 \\
--- & L 1=0 \\
-- & L 1=L 0 / 2
\end{array}
$$

(f)

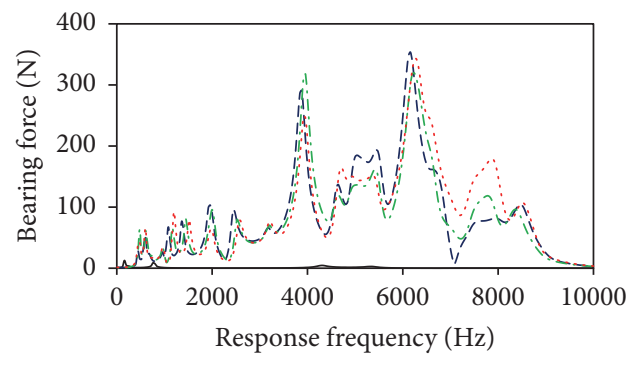

$$
\begin{array}{ll}
\text { - Pure torque } \quad \cdots \cdots & L 1=L 0 \\
--- & L 1=0 \\
-- & L 1=L 0 / 2
\end{array}
$$

(h)

FiguRE 10: Dynamic bearing force on the output side of the planet carrier shaft excited by the transmission error of (a) sun-planet 1, (b) ring-planet 1, (c) sun-planet 2, (d) ring-planet 2, (e) sun-planet 3, (f) ring-planet 3, (g) sun-planet 4, and (h) ring-planet 4. 
Based on the results presented, the following conclusions can be derived:

(i) Under combined force and moment loading condition, bias load distribution on meshing gears easily occurs, which is determined by the collinearity of the positioning of external vertical force with the bearing of the driving component significantly.

(ii) The refined natural frequencies have been revealed after considering the flexibility of all components in the proposed model, comparing with lumped dynamic models with rigid assumptions, which is helpful to explain the linkage between the resonance peaks.

(iii) Different from the previous results from pure moment loading, assuming that all planets have good consistencies, dynamic mesh forces would not be identical for all planets under combined force and moment loading conditions. The maximum peak dynamic mesh force amplitude occurs for planets whose radial position is collinear to the additional vertical force direction and most away from the imposed force position. An interesting point is that peak dynamic mesh force amplitudes are mostly reduced for meshing gears in addition to a few planets meshing with the driving gear.

(iv) The magnitude of bearing dynamic forces is expanded significantly under combined force and moment loading no matter what the positioning is. The bearing dynamic forces will propagate from there to the vehicle body through the transmission mountings and thus lead to potential vibration and noise problem.

\section{Conflicts of Interest}

The authors declare that there are no conflicts of interest regarding the publication of this article.

\section{Acknowledgments}

The work presented in this paper has been supported financially by the National Natural Science Foundation of China (Grants 51705012 and 51405010), National Aerospace Science Foundation of China (Grant no. 2015ZA51003), and Beijing Key Laboratory for High Efficient Power Transmission and System Control of New Energy Resource Vehicle. Finally, the authors would like to thank Dr. Ahmet Kahraman (Ohio State University) for his helpful materials.

\section{References}

[1] A. Kahraman, "Free torsional vibration characteristics of compound planetary gear sets," Mechanism and Machine Theory, vol. 36, no. 8, pp. 953-971, 2001.

[2] J. Lin and R. G. Parker, "Analytical characterization of the unique properties of planetary gear free vibration," Journal of Vibration and Acoustics, vol. 121, no. 3, pp. 316-321, 1999.

[3] P. Velex and L. Flamand, "Dynamic response of planetary trains to mesh parametric excitations," Journal of Mechanical Design, vol. 118, no. 1, pp. 7-14, 1996.
[4] M. Inalpolat and A. Kahraman, "A dynamic model to predict modulation sidebands of a planetary gear set having manufacturing errors," Journal of Sound and Vibration, vol. 329, no. 4, pp. 371-393, 2010.

[5] Y. Guo and R. G. Parker, "Purely rotational model and vibration modes of compound planetary gears," Mechanism and Machine Theory, vol. 45, no. 3, pp. 365-377, 2010.

[6] Z.-H. Liu, S.-J. Wu, X.-S. Wang, and B. Qian, "Nonlinear dynamics of compound planetary gear sets based on incremental harmonic balance method," Journal of Vibration and Shock, vol. 31, no. 3, pp. 117-122, 2012.

[7] M. Kubur, A. Kahraman, D. M. Zini, and K. Kienzle, "Dynamic analysis of a multi-shaft helical gear transmission by finite elements: model and experiment," Journal of Vibration and Acoustics, vol. 126, no. 3, pp. 398-406, 2004.

[8] V. Abousleiman, P. Velex, and S. Becquerelle, "Modeling of spur and helical gear planetary drives with flexible ring gears and planet carriers," Journal of Mechanical Design, vol. 129, no. 1, pp. 95-106, 2007.

[9] M. Ebenhoch, J. Greiner, and G. Gaertner, "9-speed Transmission for Front Wheel Drive Applications," in Proceedings of International VDI Congress-Drivetrain for Vehicles, pp. 57-80, Friedrichshafen, Germany, 2013.

[10] X. Xu Key, "Key Engineering Technology of Shengrui 8AT," in Proceedings of the 2011 International Symposium on Transmission Innovation and China Industrialization, Shanghai, China, 2011.

[11] T. F. Conry and A. Seireg, "A mathematical programming technique for the evaluation of load distribution and optimal modifications for gear systems," Journal of Manufacturing Science and Engineering, vol. 95, no. 4, pp. 1115-1122, 1973.

[12] D. R. Houser, "Research in the gear dynamics and gear noise research laboratory," in Proceedings of 1982 SAE International Off-Highway and Powerplant Congress and Exposition, vol. 87, pp. 53-58.

[13] H. Yue, Y. Liu, X. Xu, and J. Lai, "Study on vibration characteristics and tooth profile modification of a plus planetary gear set," Journal of Vibroengineering, vol. 16, no. 2, pp. 954-964, 2014.

[14] J. Lai, Research on the Tooth Contact Characteristic and Noise Reduction Method of Helical Gear [Master, thesis], Beihang University, Beijing, China, 2015.

[15] M. Inalpolat and A. Kahraman, "A theoretical and experimental investigation of modulation sidebands of planetary gear sets," Journal of Sound and Vibration, vol. 323, no. 3-5, pp. 677-696, 2009.

[16] A. Kahraman, "Planetary gear train dynamics," Journal of Mechanical Design, vol. 116, no. 3, pp. 713-720, 1994. 


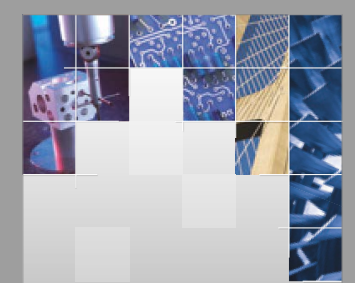

\section{Enfincering}
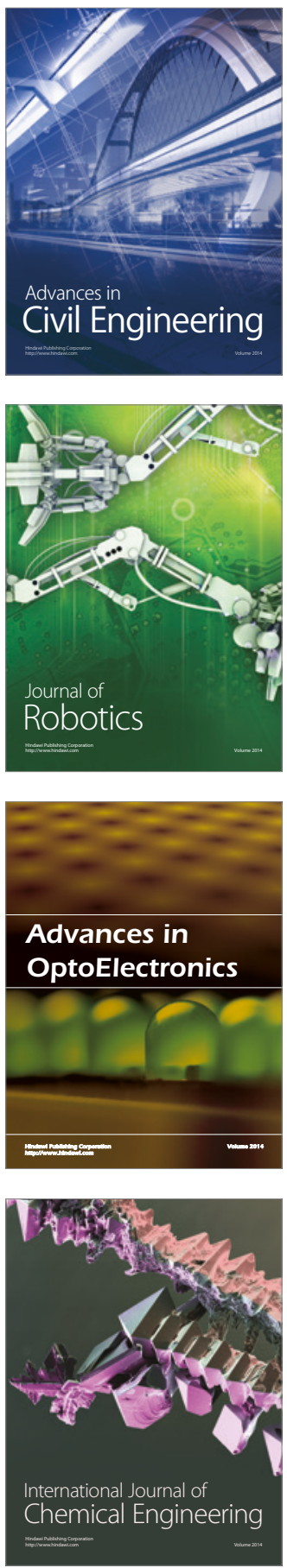

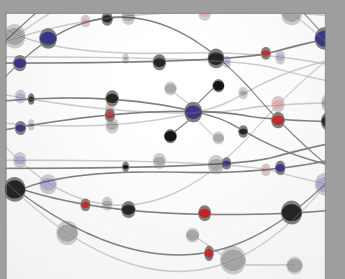

The Scientific World Journal

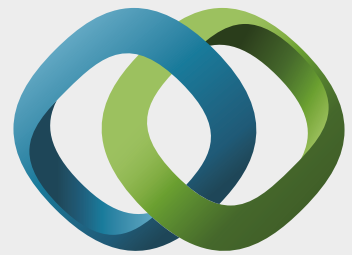

\section{Hindawi}

Submit your manuscripts at

https://www.hindawi.com
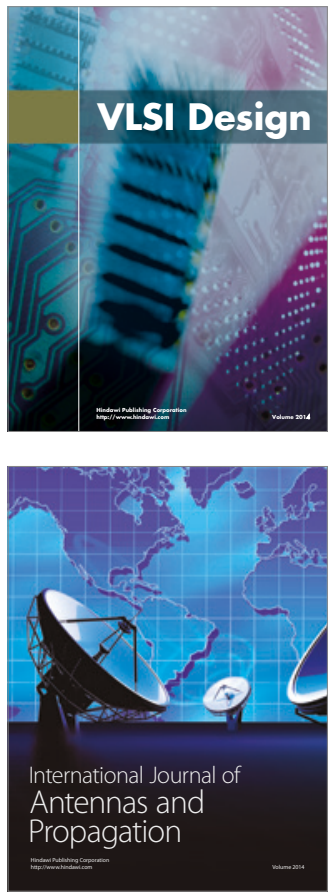

\section{Rotating}

Machinery
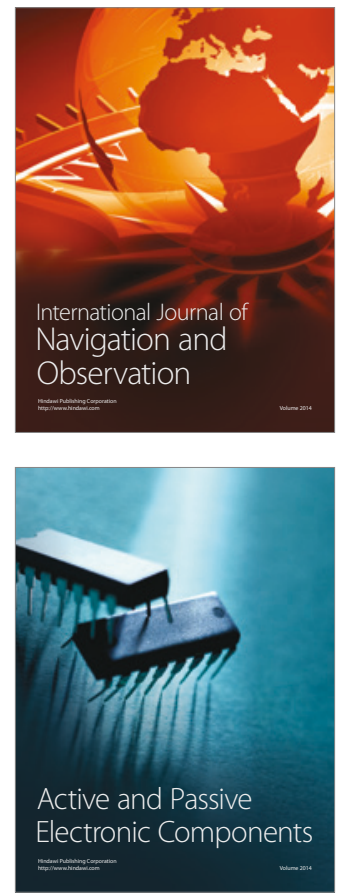
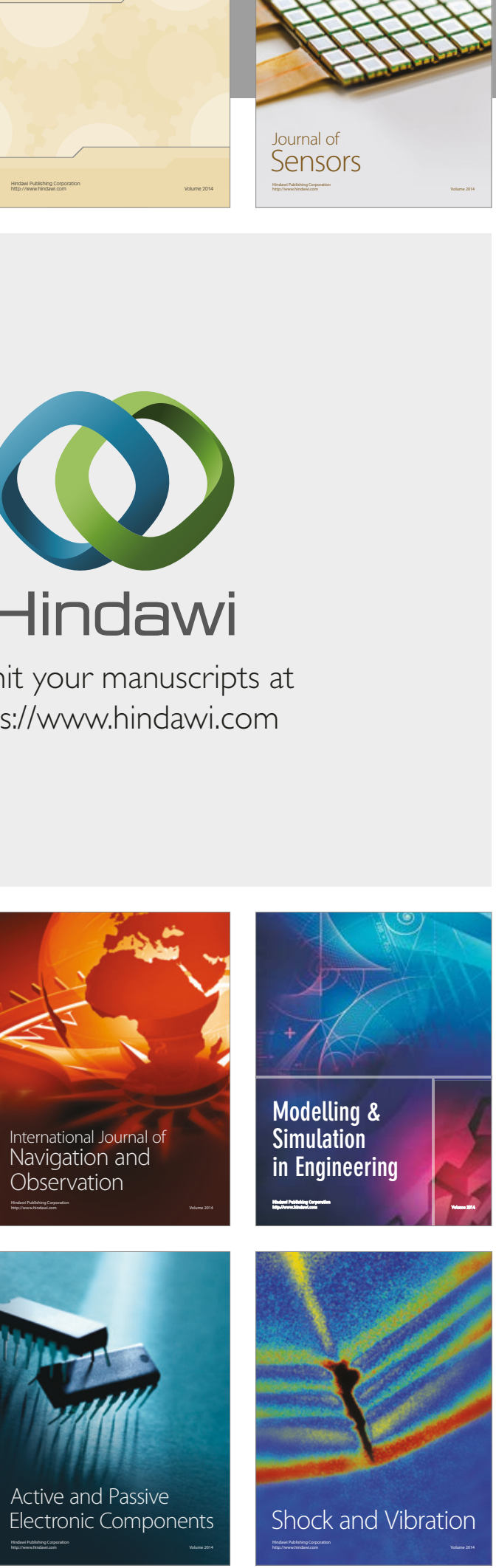
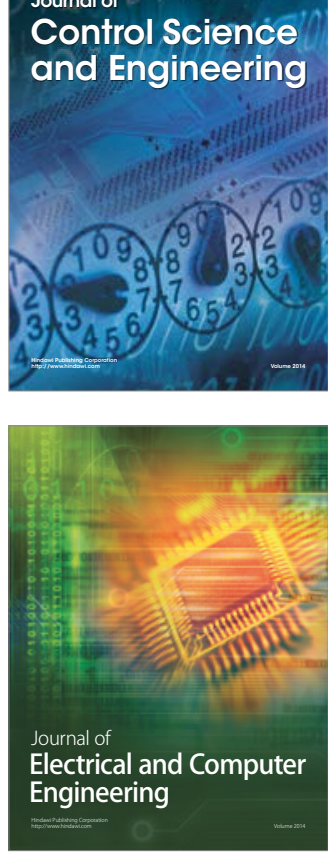

Distributed

Journal of

Control Science

and Engineering
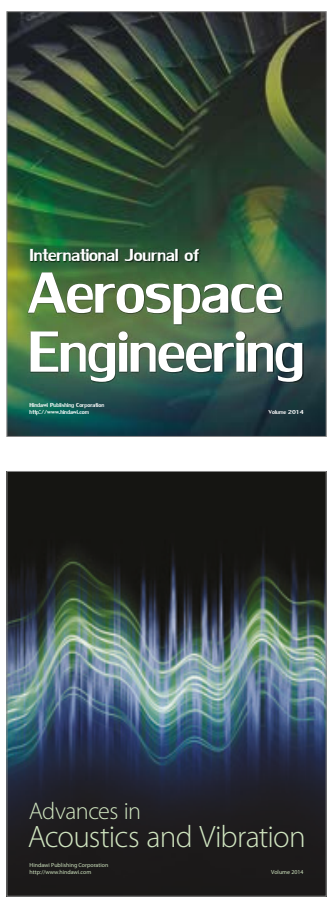

Sensor Networks 